\title{
From Basic Flow Property to MEMS Multiparameter Sensors
}

\author{
Roman Beigelbeck ${ }^{1}$, Samir Cerimovic ${ }^{1}$, Almir Talic ${ }^{1}$, Franz Kohl' ${ }^{1}$, Artur Jachimowicz ${ }^{1}$, \\ Thomas Voglhuber-Brunnmaier ${ }^{2}$, Bernhard Jakoby ${ }^{2}$, Gerald Urban $^{3}$, and Thilo Sauter ${ }^{1}$ \\ ${ }^{1}$ Center for Integrated Sensor Systems, Danube University Krems, A-2700 Wiener Neustadt, Austria, \\ roman.beigelbeck@donau-uni.ac.at \\ ${ }^{2}$ Institute for Microelectronics and Microsensors, Johannes Kepler University, A-4040 Linz, Austria \\ ${ }^{3}$ Laboratory for Sensors, IMTEK, University of Freiburg, D-79085 Freiburg, Germany
}

\begin{abstract}
Thermal sensors rely on a simple transduction principle. They exhibit the advantage of having a simple and easy-to-manufacture structure requiring no moving parts, which makes them robust, costeffective, and versatile. Consequently, they are widely used in many applications. This paper demonstrates how basic calorimetric flow and thermal parameter sensors, both based on silicon thinfilm technology, can be extended to more sophisticated devices by modifying the sensor layout or by altering the measurement method. In the context of flow sensors, we introduce direction and angular resolved devices as well as an ultra-low power and a multiport hot-film device. In terms of thermal parameter sensors, we report on two different designs to measure the thermal conductivity and diffusivity of fluids at rest. Finally, we also present two concepts of how to merge calorimetric flow sensors and thermal parameter sensors into multiparameter flow sensors which offer a self-calibrating feature irrespective of the fluid type and flow state.
\end{abstract}

Key words: calorimetric flow sensors, thermal parameter sensors, multiparameter flow sensors

\section{Introduction}

Thermal flow sensors are a highly common type of flow sensors with versatile applications. They can be found in air conditioning systems of buildings, flow cytometry, air supplies of cleanroom environments, and in meteorology for wind and storm quantification as well as in gas chromatographs, to name just a few examples [1]. The basic transduction concept of thermal flow sensors is more than 100 years old [2]. It relies on the detection of flow-induced changes of a temperature profile generated by a heating element.

Common thermal flow sensors are either based on the anemometric or calorimetric principle. In the former, the cooling of a heater element (a wire in the simplest case) in a flow is utilized to deduce the velocity of the flow. This, however, does not allow determining the direction of the flow. In case of calorimetric flow sensors (CFS), spatially separated temperature sensors are used in addition to the heater element. By arranging, e.g., two temperature sensors next to the heater in up- and downstream direction, respectively, the direction can be obtained as well.

Thermal parameter sensors (TPS) for fluids represent a different type of sensor, which, however, can be related in terms of the applied measurement technology. TPS aim at the determination of thermal material parameters, i.e., the specific thermal conductivity $\lambda$, the diffusivity $\kappa$, and the specific and volumetric thermal capacitances $c_{\mathrm{p}}$ and $c_{\mathrm{v}}$, respectively. These sensors exploit thermal diffusion and in general require that the sample fluid is at rest, where the diffusive heat transport between a heater and a temperature sensor is evaluated for particular excitation of the heater, which in turn allows determining the above mentioned parameters which affect this heat transport.

In microtechnology, the heating and temperature sensing elements of the CFS and TPS are commonly realized as thin-film structures deposited on a carrier substrate. We have been active in this field of silicon micromachined CFS and TPS for more than a quarter century now. During this period, we developed a vast number of different CFS and TPS devices that all have the following four things in common:

1. They rely on an in-house developed and over the years optimized silicon micromachining process facilitating highly sensitive thin-film thermistors made of amorphous germanium (aGe). These thermistors allow for detection of temperature changes down to $10 \mu \mathrm{K}$ at superior 
signal-to-noise ratios and exhibit a relatively large temperature coefficient of resistance (TCR) in the range of $-2 \% / K$. The high specific resistivity (about $5 \Omega \mathrm{m}$ ) of aGe facilitates low operating currents enabling small-sized interconnecting lines of low parasitic thermal conductances. However, this technology features one serious drawback: It limits the temperatures of all following fabrication processes to below $\sim 150^{\circ} \mathrm{C}$. Above this threshold, the aGe would start to recrystallize resulting in a loss of the aforementioned outstanding properties.

2. Chromium has been chosen as material for the thin-film heater owing to its low TCR.

3. All heating and temperature sensing elements are either embedded in a thin-film diaphragm or in several spatially separated thin-film microbridges, both made of $\mathrm{Si}_{x} \mathrm{~N}_{y}-\mathrm{SiO}_{2}$ with a typical thickness between 1-1.4 $\mu \mathrm{m}$. The extremely low thermal mass of these thin-film structures facilitates an efficient thermal decoupling between active sensing region and bulk chip, resulting in high sensitivities of the sensor device and fast response at the same time.

4. Additional substrate thermistors (labeled by ST in the figures below) located on the silicon rim provide the ability to monitor the chip temperature which is usually very close to the ambient temperature $T_{0}$.

In this contribution, we showcase representative examples of previously devised micromachined CFS and TPS embodiments and discuss their pros and cons. Finally, both concepts are merged into multiparameter flow sensors (MPFS) in order to obtain miniaturized, self-calibration ready CFS or TPS for fluids in motion.

\section{Micromachined Calorimetric Flow Sensors}

\section{Classic CFS Layout}

Figure 1 depicts a photomicrograph of the simplest CFS layout. Around a resistive heater made of $\mathrm{Cr}$, two aGe thermistors denoted by $\mathrm{DT}_{1}$ and $\mathrm{DT}_{2}$ are symmetrically arranged [3]. In sensor operation, the heater is subjected to a DC current. Without flow, the temperature profile is symmetrically distributed around the heater and thus $\mathrm{DT}_{1}$ and $\mathrm{DT}_{2}$ measure the same temperature values. Under flow conditions, this symmetry is broken. For incompressible flows, the combined conductive and convective heat transport induced by the passing fluid is governed by the heat transfer equation

$$
\nabla^{2} \vartheta-\frac{1}{\kappa}\left[\mathrm{v} \cdot \nabla \vartheta+\frac{\partial \vartheta}{\partial t}\right]=Q
$$

where $\nabla, \mathrm{v}, t$, and $Q$ denote the del operator, flow velocity vector, time, and heat source, respectively. The local excess temperature $\vartheta$ in the fluid is related to the local temperature $T$ by $\vartheta=T-T_{0}$. Measuring the velocity dependent excess temperatures $\vartheta(v)$ at the up- and downstream thermistor allows for determination of flow quantities like flow velocity, flow amount, and flow direction.

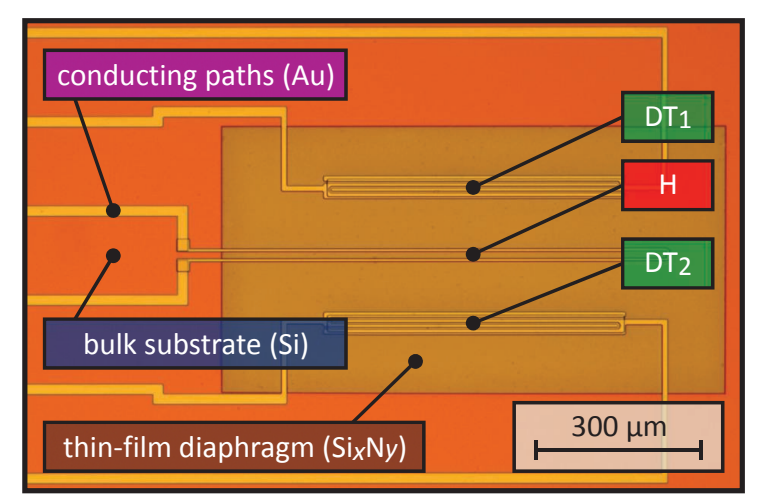

Figure 1: Classic CFS layout

Figure 2 depicts a typical transduction characteristic of the temperature difference between up- and downstream thermistor of such a CFS. At lower flow rates, the change in the temperature is high resulting in a superior sensitivity in this mode of operation. Above a maximum, the characteristic decreases and the flow rate versus temperature characteristics become ambiguous. The flow sensitivity of the CFS in this range is rather low and therefore this mode of operation should preferably be avoided.

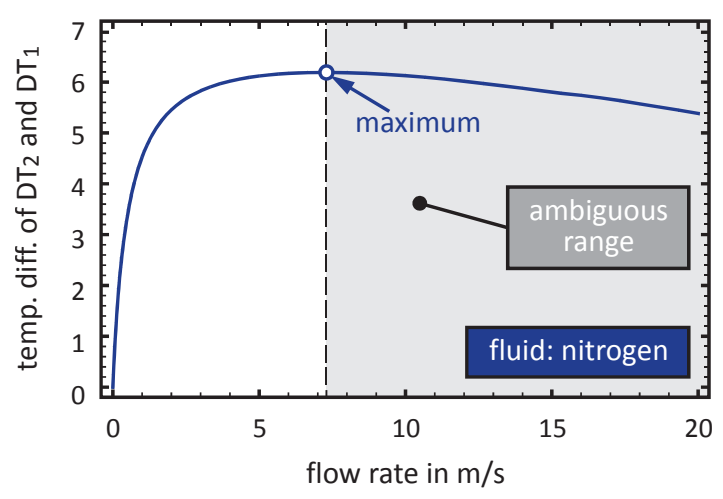

Figure 2: Typical CFS transduction characteristic

\section{Ultra-Low Power Design}

The power consumption of the classic CFS layout is typically in the range of a few $\mathrm{mW}$. However, certain applications such as autonomous sensor swarms require devices with far 
less power consumption. For this reason, the sensor design outlined in Figure 3 was created [4]. Its working principle is based on the accurate measurement of the self heating under the cooling by the fluid flow passing the highly sensitive aGe thermistors, so that they work simultaneously as heating and temperature sensing elements. Exploiting the temperature field induced and measured by the thermistors $\mathrm{DT}_{1-4}$ results in a combination of anemometric and calorimetric heat transduction.

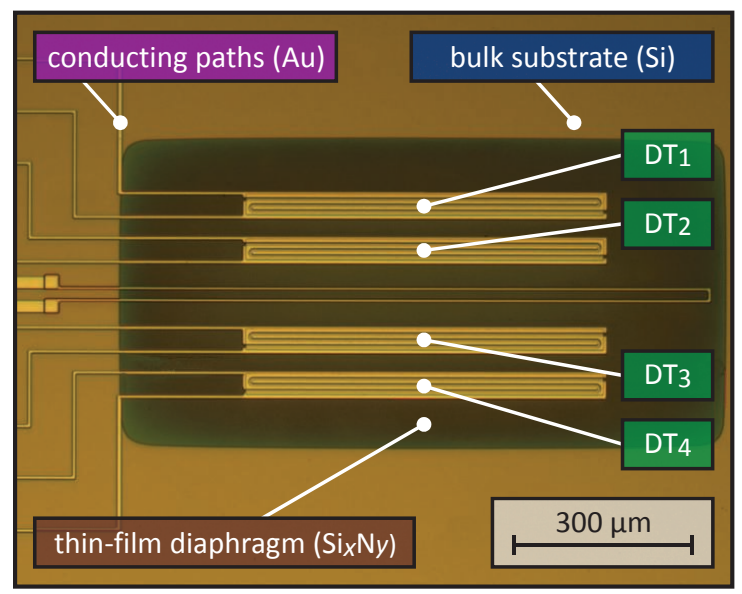

Figure 3: Ultra-low power design

In sensor operation, the four thermistors are connected to a Wheatstone bridge, where $\mathrm{DT}_{2}$ and $\mathrm{DT}_{3}$ form one bridge leg while $\mathrm{DT}_{1}$ and $\mathrm{DT}_{4}$ yield the second bridge leg. This bridge is controlled by a current source which leads to self-heating of the four thermistors. In the flowfree case, the generated temperature profile is symmetrical with respect to the diaphragm center if all thermistors exhibit the same resistance. In this case, the bridge is in the balanced state and the bridge detuning voltage disappears. However, if a flow is imposed, then convective heat transport by the fluid distorts the temperature profile and the bridge becomes detuned. Both the bridge detuning voltage and the voltage across the supply terminals of the bridge can be used to derive the flow velocity. Due to the high temperature resolution of the aGe thermistors, very low heating currents may be used, forcing the total power consumption of the sensor into the sub-mW range. Pulsed operation can be used to further reduce the power consumption.

\section{Multiport Hot-Film Design}

As stated above, the anemometric hot-film principle does not allow for the determination of the direction of fluid flow, but it offers an unambiguous transduction characteristic. With calorimetric sensors, the determination of the flow direction is possible, however, due to their measurement principle an ambiguous transduction characteristic occurs, limiting the useful measurement range. In order to circumvent both drawbacks, the sensor design of Figure 4 was created, where a single aGe hot film is contacted by five metal strips and connected analogous to the ultra-low power design to form a Wheatstone bridge [5].

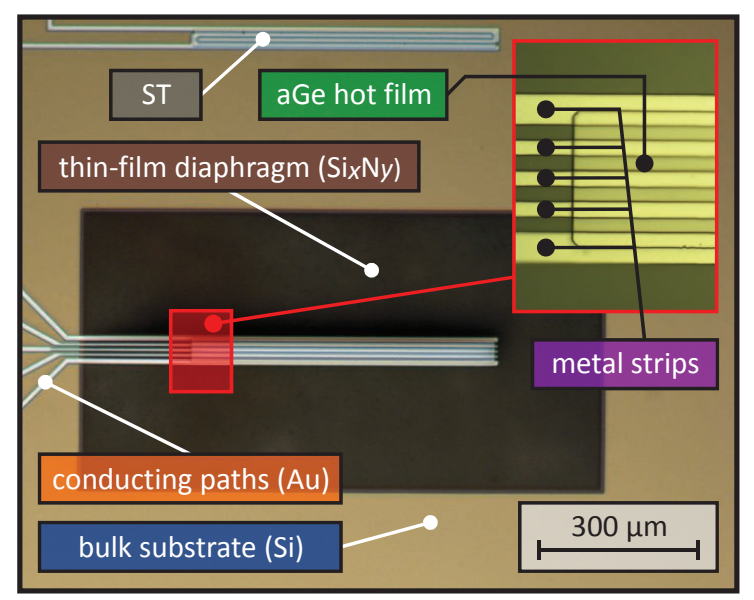

Figure 4: Multiport hot-film design

\section{Wind Sensor Design}

The wind sensor design of Figure 5 combines the pronounced bidirectional characteristic of several classic CFS into a device that resolves not only the flow magnitude, but also the flow direction in 2D [6]. In the center of the diaphragm, a DC controlled heating element $\mathrm{H}$ generates a symmetric temperature profile in the flow-free case. Hence, all thermistors DT $_{1-8}$ measure the same temperature. Flows along a specific angular direction distort the rotational symmetry of the temperature profile which can be measured using the $\mathrm{DT}_{1-8}$ temperatures. The flow velocity and the flow angle can be computed from these data.

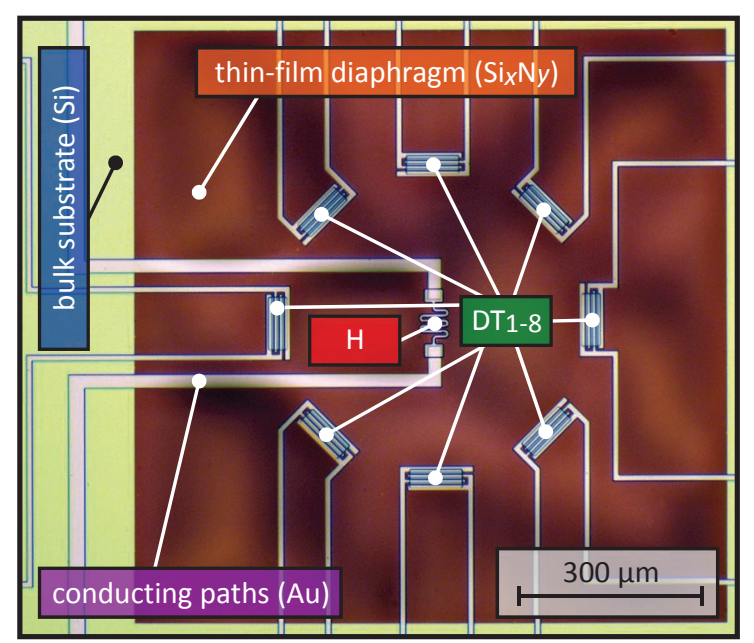

Figure 5: Wind sensor design 
Figure 6 shows representative directional characteristics of the flow-induced temperature changes of opposite thermistors [6].

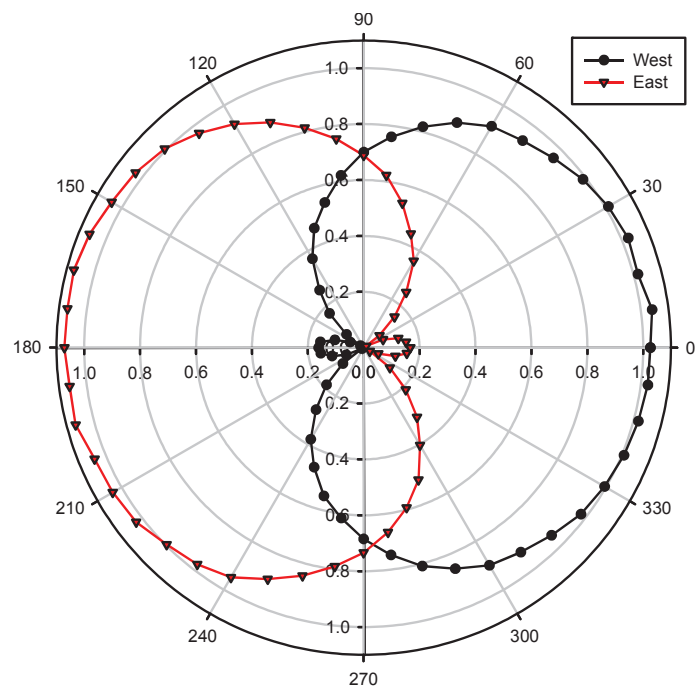

Figure 6: Directional characteristics of a wind sensor

\section{Micromachined Thermal Parameter Sensors}

Widely used TPS feature distinct differences to thermal flow sensors. First, they commonly require that the fluid is at rest such that the diffusive heat transport, which is used as a measurement principle, is not superposed by spurious convective heat transport. Secondly, a time-harmonic excitation is used, generating a diffusive heat transfer process. The sensor design of Figure 1 has been utilized as TPS of the first generation. During operation, the device is immersed into the resting fluid to be measured. Then the heater $\mathrm{H}$ is driven by a sinusoidal voltage resulting in diffusive heat propagation in the fluid that is determined by the thermal parameters of the fluid. By measuring the AC-response at $\mathrm{DT}_{1-2}, \lambda$ and $\kappa$ can be evaluated from the measured amplitude and phase. Based on a numerical model, the nomogram shown in Figure 7 has been established which facilitates the extraction of these parameters from the measured values [7]. However, this sensor design suffers from two drawbacks. They are both related to the unavoidable heat conduction in the thin-film diaphragm which competes with the conductive heat path through the measured fluid. First, when measuring low conducting fluids, this shunt impairs the measurement reliability. Secondly, the heat shunt impedes a simple analytical modeling of the sensor functionality as well as an easy parameter extraction from the raw measurement data. In order to circumvent both drawbacks, the structure depicted in Figure 8 has been developed [8].

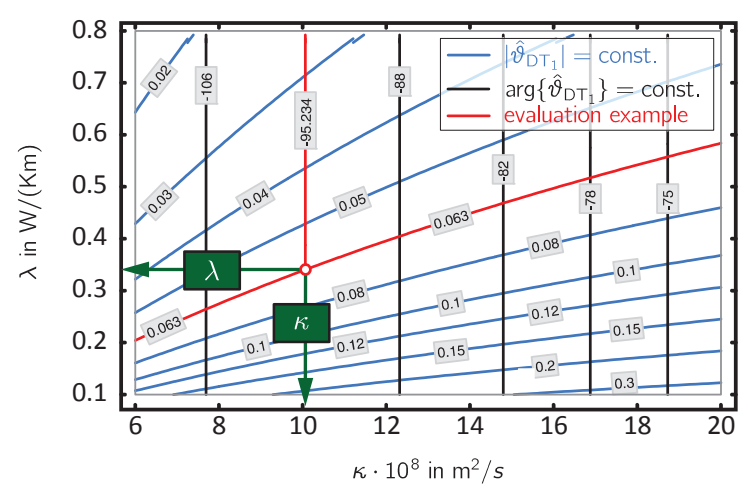

Figure 7: Nomogram-based evaluation of the thermal parameters of the fluid. The red characteristic illustrates an evaluation example, where the measured amplitude (in Kelvin) and phase (in degrees) characteristics intersect yielding $\lambda$ and $\kappa$.

In contrast to the previously described TPS diaphragm embodiment, the multibridge structure in Figure 8 consists of three individual microbridges where each bridge either carries a resistive heater $\mathrm{H}$ or an aGe thermistor $\mathrm{BT}_{1-2}$. The bridges are manufactured by an additional fabrication step in which the diaphragm is patterned from the front side using photolithography and punched by a following reactive ion etching process.

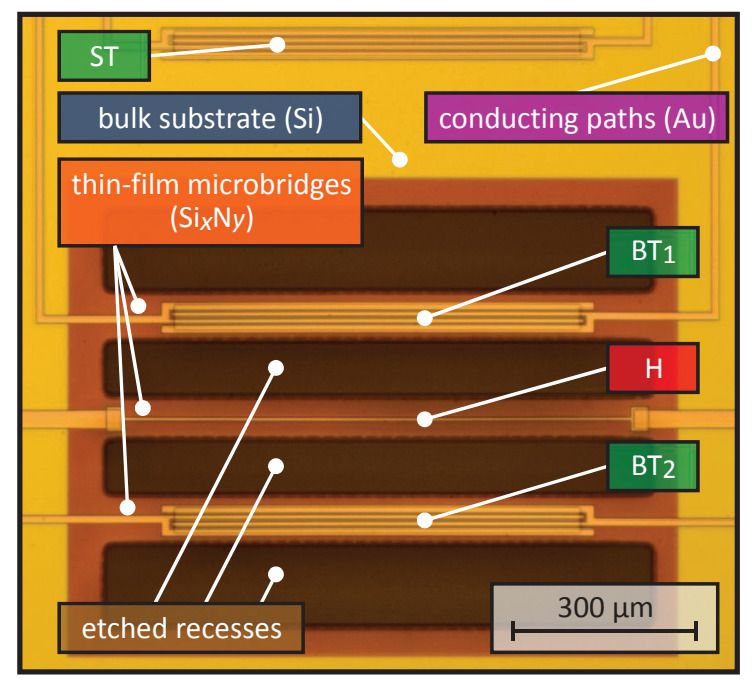

Figure 8: Microbridge-based TPS design

Compared to the diaphragm structures, this design reduces the spurious heat shut between heater $\mathrm{H}$ and thermistors $\mathrm{BT}_{1-2}$ to a minimum and thus allows the application of a simple analytical model for analyzing the heat transfer within the fluid. The complex-valued frequency response of the peak excess temperature at the thermistor site is governed by

$$
\hat{\vartheta}(f)=\frac{P}{l} \frac{1}{2 \pi \lambda} \mathrm{K}_{0}\left(\sqrt{\frac{\mathrm{j} 2 \pi f d^{2}}{\kappa}}\right),
$$


where $\mathrm{j}=(-1)^{1 / 2}$ and $f, P, l, d$, and $\mathrm{K}_{0}[\cdot]$ denote the frequency of the heat oscillation, the imposed heat, the length of the heater, the mean distance between heater and thermistor, and the modified Bessel function of the second kind and zeroth-order, respectively. Asymptotic expansion of the first derivative of $\ln |\hat{\vartheta}(f)|$ with respect to $(f)^{1 / 2}$ for large arguments of $K_{0}[\cdot]$ reveals that this function asymptotically approaches a straight line with a slope depending only on $\kappa$ but not on $\lambda$. This property can be exploited for the immediate evaluation of the thermal diffusivity from the measured excess frequency response (see Figure 9). Afterwards, rearranging and applying Eq. (2) to an arbitrary point on the measured frequency response allows for direct calculation of $\lambda$ [8].

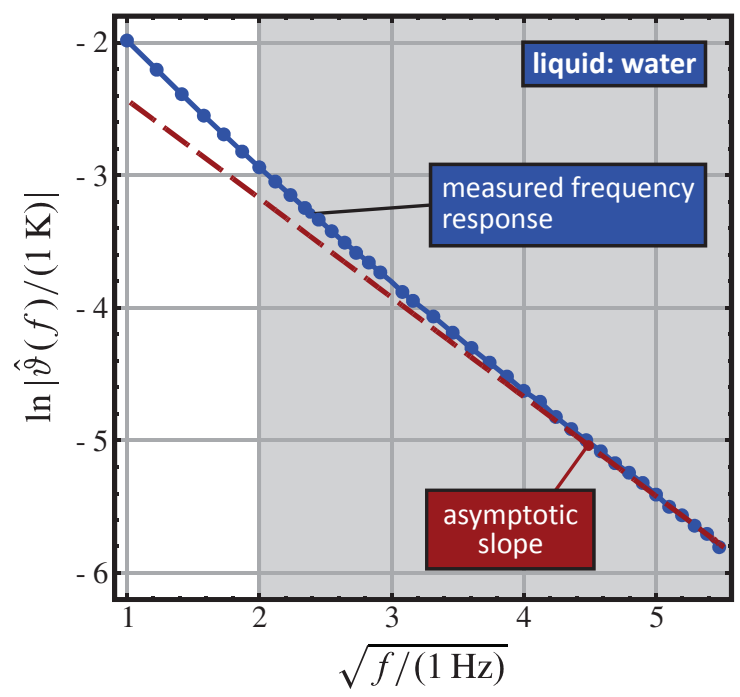

Figure 9 Determination of the asymptotic slope of the excess temperature frequency response at $\mathrm{BT}_{1}$ from which $\kappa$ and $\lambda$ can directly be calculated.

\section{Micromachined Multiparameter Flow Sensors}

All previously introduced CFS concepts exhibit the disadvantage that they require calibration procedures whenever the composition of the fluid changes or the thermophysical properties of the fluid vary due to changes in pressure or temperature. By contrast, the aforementioned TPS concepts are based on conductive heat transfer and thus are not suitable for fluids in motion. Both limitations can be overcome with the same type of sensors, the so-called multiparameter flow sensors (MPFS) yielding self-calibration ready CFS on the one hand and TPS for flowing fluids on the other hand.

Figure 10 depicts an MPFS design [9]. It basically consists of a circular multilayer thinfilm diaphragm comprising six thin-film elements. The circular chromium heater $\mathrm{H}$ is placed around the center of the diaphragm and closely surrounds a central aGe thermistor CT. Its periphery is split up into four equal arcshaped segments. The gaps between the arcs allow for the interconnection of CT. The remaining four thermistors $\mathrm{DT}_{1-4}$ are concentrically arranged around $\mathrm{H}$. In default sensor operation, the heater is simultaneously driven in $A C$ and $D C$ mode. The measuring principle utilizes high frequency heat generation at $200 \mathrm{~Hz}$ in order to confine the AC heat transfer to a thin region above the surface of the channel wall, where the flow velocity profile is always close to zero such that evaluation methods for TPS can be applied. From the associated $\mathrm{AC}$ temperature response at $\mathrm{CT}$ the thermal fluid parameters can be extracted, while the $\mathrm{DC}$ part at $\mathrm{DT}_{1-4}$ yields information about the flow quantities (i.e., flow direction, flow velocity, and flow amount) of the fluid passing the sensor's surface, both at flow condition.

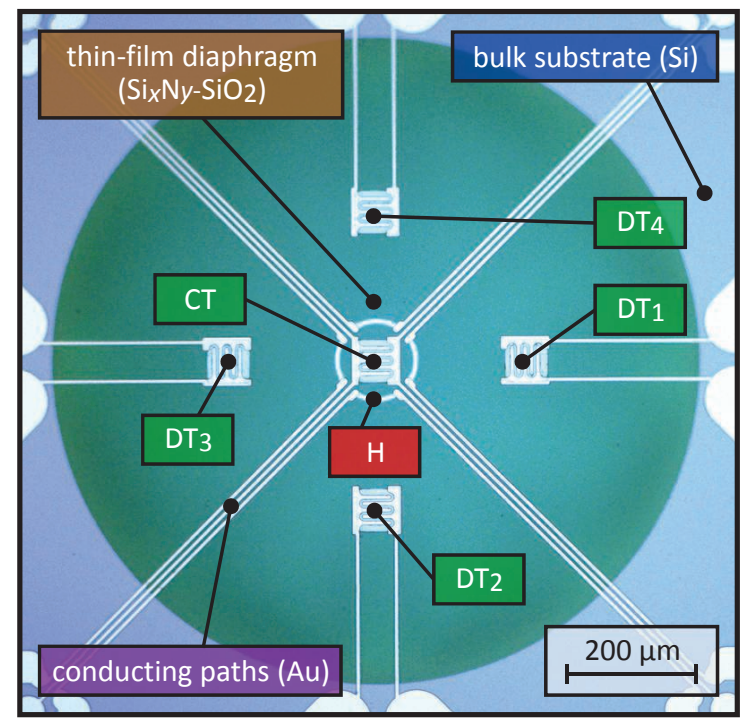

Figure 10: Multiparameter wind sensor

Figure 11 shows another concept for an MPFS suitable for measurement in gases, which comprises two separated wind sensor devices. The setup implements a "flow shelter". In case of one of the sensors, again holes are etched into the diaphragm (see Figure 11a). Then the device is flipped and bonded to another one which features no holes in the diaphragm (see Figure 11b). This sandwich structure is then mounted into a flow channel such that the diaphragm with the holes is in plane with the channel walls (see Figure 11c). During operation (i.e., flow in the channel), the fluid will intrude through the holes and fill the cavity area by diffusion where virtually no flow will be present in the lower area of the cavity. By means of simulations, the geometry was 
optimized to minimize the spurious flow above the lower sensor such that the previously described TPS concepts can be applied to determine the thermal fluid parameters. These values can then be used to calibrate the measurements obtained from the upper sensor measuring the flow in the channel. In that way, a sensor device capable of self-calibration can be realized.
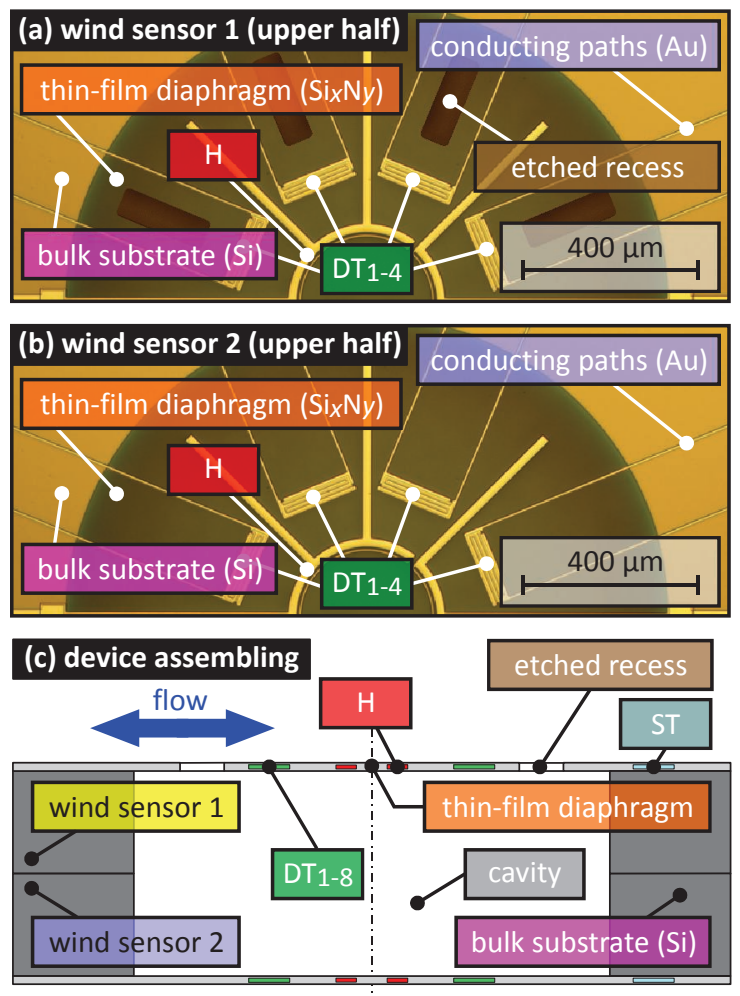

Figure 11: Self-calibration ready wind sensor concept comprising a resistive heater $\mathrm{H}$ and eight aGe thermistors $\mathrm{DT}_{1-8}$. (a) Upper half of the $360^{\circ}$ wind sensor chip featuring etched recesses in the diaphragm. (b) Upper half of the $360^{\circ}$ wind sensor chip without recesses. (c) Assembling of both devices the self-calibration ready MPFS.

\section{Conclusion}

This paper demonstrated the versatility of the simple thermal flow transduction concept. Starting from the basic layout with a central heater and two thermistors on a membrane, sensors with varying degree of sophistication can be implemented by basically changing the layout. We presented direction-sensitive as well as ultra-low power flow sensors, but also sensors suited for determining the essential thermal parameters of a resting fluid. Combining these two principles will even allow for the design of sensors that will be selfcalibrating and will be able to identify the fluid and its parameters during normal operation, which will be a major step towards robust and easy-to-use flow sensors.

\section{Acknowledgements}

The Center for Integrated Sensor Systems gratefully acknowledges partial financial support by the European Regional Development Fund (ERDF) and the province of Lower Austria.

\section{References}

[1] J. T. W. Kuo, L. Yu, and E. Meng, "Micromachined Thermal Flow Sensors - A Review", Micromachines, vol. 3, no. 3, pp. 550-573, July 2012.

[2] G. Comte-Bellot, "Hot-wire Anemometry", Annual Review of Fluid Mechanics, vol. 9, no. 1, pp. 209-231, 1976.

[3] F. Kohl, R. Fasching, F. Keplinger, R. Chabicovsky, A. Jachimowicz, and G. Urban, "Development of miniaturized semiconductor flow sensors", Measurement, vol. 33, no. 2, pp. 109-119, 2003.

[4] S. Cerimovic, A. Talic, T. Sauter, F. Kohl, R. Beigelbeck, J. Schalko, and A. Jachimowicz, "A Novel Thermal Transduction Method for sub-mW Flow Sensors". In Proceedings of the 8th IEEE Conference on Sensors, pp. 1325-1328, Christchurch, New Zealand, October 2009.

[5] S. Cerimovic, A. Talic, R. Beigelbeck H. Antlinger, T. Sauter, J. Nicolics, B. Jakoby, and F. Keplinger, "Bidirectional micromachined flow sensor featuring a hot film made of amorphous germanium", Measurement Science and Technology, vol. 24, no. 8, pp. 084002.1-084002.16, August 2013.

[6] F. Keplinger, R. Beigelbeck, F. Kohl, J. Kuntner, A. Jachimowicz, and B. Jakoby, "High Performance Sensor for Angular Resolved Flow Measurement'. In Transducers'07: The 14th International Conference on Solid-State Sensors, Actuators and Microsystems, vol. 2, pp. 2337-2340, Lyon, France, June 2007.

[7] J. Kuntner, F. Kohl, and B. Jakoby, "Simultaneous thermal conductivity and diffusivity sensing in liquids using a micromachined device", Sensors and Actuators A, vol. 130-131, pp. 62-67, 2006.

[8] R. Beigelbeck, H. Nachtnebel, H., F. Kohl, and B. Jakoby, "A novel measurement method for the thermal properties of liquids by utilizing a bridge-based micromachined sensor", Measurement Science and Technology, vol. 22, no. 10, pp. 105407.1105407.9, August 2011.

[9] D. Reyes-Romero, K. Kogan, A. Cubukcu, and G. Urban, "Simultaneous flow and thermal conductivity measurement of gases utilizing a calorimetric flow sensor", Sensors and Actuators A: Physical, vol. 203, pp. 225-233, 2013. 